\title{
Syndrome inflammatoire multisystémique chez les enfants au Canada
}

\author{
Meghan Laverty ${ }^{1}$, Marina Salvadori', Susan G Squires ${ }^{1}$, May Ahmed ${ }^{2}$, Lisa Eisenbeis ${ }^{3}$, Santina Lee ${ }^{4}$, \\ Annick Des Cormiers ${ }^{5}$, Y Anita Li ${ }^{1 *}$
}

\section{Résumé}

Cet article présente un résumé de l'épidémiologie des cas de syndrome inflammatoire multisystémique chez les enfants (MIS-C) déclarés à l'échelle nationale au Canada par les autorités sanitaires provinciales et territoriales. Le syndrome inflammatoire multisystémique chez les enfants est un syndrome inflammatoire post-viral qui suit la maladie à coronavirus 2019 (COVID-19). Les symptômes peuvent comprendre de la fièvre, des douleurs abdominales, des vomissements, de la diarrhée, des éruptions cutanées et d'autres signes d'inflammation. Au Canada, le MIS-C est rare, avec 269 cas signalés à l'Agence de la santé publique du Canada entre le 11 mars 2020 et le 2 octobre 2021. Cent quarante-deux (53\%) de ces cas étaient des cas de COVID-19 confirmés en laboratoire ou liés épidémiologiquement à des cas de COVID-19. Des cas ont été signalés chez des nourrissons d'à peine une semaine et des jeunes de 18 ans, l'âge médian étant de six ans. Les cas étaient plus susceptibles de se produire chez les garçons que chez les filles ( $58 \%$ contre $42 \%$, respectivement; $p=0,006$ ). Presque tous les cas de MIS-C (99\%) ont nécessité une hospitalisation et $36 \%$ une admission en unité de soins intensifs. Aucun décès n'a été signalé à ce jour. La tendance temporelle du MIS-C s'aligne sur la tendance temporelle du taux d'incidence du COVID-19 rapporté chez les enfants, avec un décalage de deux à six semaines.
Cette oeuvre est mise à la disposition selon les termes de la licence internationale Creative Commons Attribution 4.0

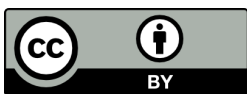

Affiliations

${ }^{1}$ Centre de l'immunisation et des maladies respiratoires infectieuses, Agence de la santé publique du Canada, Ottawa, ON ${ }^{2}$ BC Centre for Disease Control, Vancouver, BC

${ }^{3}$ Alberta Health Services, Edmonton, $A B$

${ }^{4}$ Contrôle des maladies transmissibles, Santé publique, Santé Manitoba, Winnipeg, MB

${ }^{5}$ Ministère de la Santé et des Services sociaux, Québec, QC

\section{*Correspondance :}

yanita.li@phac-aspc.gc.ca

Citation proposée : Laverty M, Salvadori MI, Squires SG, Ahmed MA, Eisenbeis L, Lee SJ, Des Cormiers A., Li YA. Syndrome inflammatoire multisystémique chez les enfants au Canada. Relevé des maladies transmissibles au Canada 2021;47(11):512-6. https://doi.org/10.14745/ccdr.v47i11a03f

Mots-clés : syndrome inflammatoire, COVID-19, santé des enfants, surveillance, MIS-C

\section{Introduction}

Depuis l'émergence du coronavirus du syndrome respiratoire aigu sévère 2 (SRAS-CoV-2) à l'origine de la maladie à coronavirus 2019 (COVID-19), les données sur les enfants et les jeunes âgés de 19 ans et moins infectés par le SRAS-CoV-2 indiquent qu'ils présentent généralement une maladie légère avec des résultats moins graves par rapport aux adultes. Cependant, le 26 avril 2020, des cliniciens du Royaume-Uni ont signalé une augmentation du nombre de comptes d'enfants, auparavant en bonne santé, présentant un syndrome inflammatoire grave avec des caractéristiques similaires au syndrome du choc toxique et à la maladie de Kawasaki incomplète (1). Ces cas sont survenus chez des enfants qui ont reçu un résultat de test positif pour une infection récente ou actuelle par le SRAS-CoV-2 ou qui avaient un lien épidémiologique avec un cas de COVID-19 (1). Depuis lors, d'autres cas d'enfants présentant un syndrome inflammatoire sévère avec des signes d'infection par la COVID-19 ont été signalés dans le monde entier. Cette maladie a été nommée syndrome inflammatoire multisystémique chez les enfants
(MIS-C) par les Centers for Disease Control and Prevention et I'Organisation mondiale de la Santé (OMS), et est définie par I'OMS comme suit (2) :

Enfants et adolescents âgés de 0 à 19 ans ayant de la fièvre depuis trois jours ou plus

ET

Deux des éléments suivants :

- Éruption cutanée ou conjonctivite bilatérale non purulente ou signes d'inflammation muco-cutanée (bouche, mains ou pieds)

- Hypotension ou choc

- Caractéristiques de dysfonctionnement myocardique, de péricardite, de valvulite ou d'anomalies des artères coronaires (y compris résultats d'ECHO ou taux élevé de Troponine/taux plasmatiques du peptide cérébral natriurétique (NT-proBNP) 
- Preuve de coagulopathie (par le temps de prothrombine, le temps de thromboplastine partielle, un taux élevé de D-dimères)

- Problèmes gastro-intestinaux aigus (diarrhée, vomissements ou douleurs abdominales)

ET

Marqueurs d'inflammation élevés tels que la protéine C-réactive, la vitesse de sédimentation des érythrocytes ou la procalcitonine

\section{ET}

Aucune autre cause microbienne évidente d'inflammation, y compris septicémie bactérienne, syndromes de choc staphylococcique ou streptococcique

\section{ET}

Preuve de la COVID-19 (réaction en chaîne par polymérase à transcription inverse (RT-PCR), test d'antigène ou sérologie positive), ou contact probable avec des patients atteints de COVID-19

Le Canada a élargi cette définition de cas pour inclure les cas répondant aux critères de I'OMS pour le MIS-C, avec et sans diagnostic COVID-19. Cela a été fait pour inclure les cas possibles qui ont pu avoir un test RT-PCR trop tard dans leur évolution de l'infection (faux négatifs), et ceux qui, plus fréquemment dans les premiers stades de la pandémie, peuvent ne pas avoir eu accès au test de dépistage de la COVID-19 ou au test sérologique (3).

L'Agence de la santé publique du Canada a commencé à recueillir des données le 30 juin 2020 sur les cas de MIS-C remontant au 11 mars 2020, date à laquelle la pandémie a été déclarée pour la première fois. Ce rapport présente les cas dont la maladie est apparue du 11 mars 2020 au 2 octobre 2021 (semaine épidémiologique 11 de 2020 à semaine 39 de 2021).

\section{Situation actuelle}

Au total, 269 cas de MIS-C ont été signalés à l'Agence de la santé publique du Canada pendant la période de surveillance. Les données du 11 mars 2020 au 31 mai 2021 étaient disponibles dans 12 des 13 provinces et territoires, dont 11 ont déclaré des cas confirmés en laboratoire, liés épidémiologiquement et non liés à la COVID-19 et un a déclaré uniquement des cas confirmés en laboratoire. Les données de 11 provinces et territoires étaient disponibles pour le reste de la période de surveillance. Sur les 269 cas, 127 (47 \%) ont reçu des résultats de tests positifs pour la COVID-19 par RT-PCR, test antigénique ou sérologie et 15 autres (6\%) ont été épidémiologiquement liés à un cas de COVID-19 confirmé en laboratoire. Les 127 autres (47\%) ont reçu des résultats de tests ou n'ont pas passé de test pour la COVID-19. Les détails sur les tests de dépistage de la COVID-19 effectués pour chaque cas n'étaient pas disponibles. La proportion de cas de MIS-C parmi les cas confirmés de COVID-19 chez les enfants âgés de 19 ans et moins était de 0,039\% au Canada pendant la période de surveillance.

Les caractéristiques des cas de MIS-C rapportés au Canada sont résumées dans le tableau 1. L'âge médian était de six ans (intervalle d'une semaine à 18 ans), et $58 \%$ des cas concernaient des enfants âgés de cinq ans et plus. Lorsque les cas sont limités à ceux dont le test de dépistage de la COVID-19 est positif ou qui ont un lien épidémiologique avec un cas confirmé de COVID-19, I'âge médian est de huit ans (intervalle d'une semaine à 18 ans), $70 \%$ des cas étant âgés de cinq ans ou plus. Cela diffère de la maladie de Kawasaki, qui touche principalement les enfants de moins de cinq ans (4). Le syndrome inflammatoire multisystémique chez les enfants était plus susceptible de se produire chez les garçons que chez les filles $(p=0,006)$, plus de la moitié (58\%) des cas signalés étant des garçons. La quasi-totalité (99\%) des cas de MIS-C ont nécessité une hospitalisation, $36 \%$ d'entre eux nécessitant une admission en unité de soins intensifs. Lorsque des renseignements sur les résultats étaient disponibles, la majorité des cas s'étaient rétablis. Les autres cas étaient soit en convalescence, soit stables au moment de la mise à jour la plus récente du rapport de cas.

Aucun décès n'a été signalé à ce jour.

\section{Tableau 1 : Caractéristiques des cas signalés de syndrome inflammatoire multisystémique chez les enfants selon le statut d'infection par le SRAS-CoV-2a, Canada, 11 mars 2020 au 2 octobre 2021}

\begin{tabular}{|c|c|c|c|c|c|c|c|c|}
\hline \multirow[t]{2}{*}{ Caractéristiques } & \multicolumn{2}{|c|}{$\begin{array}{l}\text { Confirmation en } \\
\text { laboratoire uniquement } \\
(n=127)\end{array}$} & \multicolumn{2}{|c|}{$\begin{array}{l}\text { Lien confirmé en } \\
\text { laboratoire et/ou } \\
\text { épidémiologique } \\
\qquad(n=142)\end{array}$} & \multicolumn{2}{|c|}{$\begin{array}{l}\text { Aucune preuve } \\
\text { connue d'infection ou } \\
\text { d'exposition au } \\
\text { SRAS-CoV-2 } \\
(n=127)\end{array}$} & \multicolumn{2}{|c|}{$\begin{array}{l}\text { Tous les patients } \\
\qquad(\mathrm{n}=269)\end{array}$} \\
\hline & $\mathbf{n}$ & $\%^{b}$ & $\mathbf{n}$ & $\%$ & $n$ & $\%$ & $\mathbf{n}$ & $\%$ \\
\hline \multicolumn{9}{|l|}{ Sexe } \\
\hline Garçon & 82 & 65 & 92 & 65 & 65 & 51 & 157 & 58 \\
\hline Fille & 45 & 35 & 50 & 35 & 62 & 49 & 112 & 42 \\
\hline \multicolumn{9}{|c|}{ Catégorie d'âge (années) } \\
\hline Moins d'un an & 1 & 1 & 1 & 1 & 15 & 12 & 16 & 6 \\
\hline 1 à 4 & 36 & 28 & 41 & 29 & 55 & 43 & 96 & 36 \\
\hline
\end{tabular}


Tableau 1 : Caractéristiques des cas signalés de syndrome inflammatoire multisystémique chez les enfants selon le statut d'infection par le SRAS-CoV-2a, Canada, 11 mars 2020 au 2 octobre 2021 (suite)

\begin{tabular}{|c|c|c|c|c|c|c|c|c|}
\hline \multirow[t]{2}{*}{ Caractéristiques } & \multicolumn{2}{|c|}{$\begin{array}{l}\text { Confirmation en } \\
\text { laboratoire uniquement } \\
\qquad(n=127)\end{array}$} & \multicolumn{2}{|c|}{$\begin{array}{l}\text { Lien confirmé en } \\
\text { laboratoire et/ou } \\
\text { épidémiologique } \\
\qquad(n=142)\end{array}$} & \multicolumn{2}{|c|}{$\begin{array}{l}\text { Aucune preuve } \\
\text { connue d'infection ou } \\
\text { d'exposition au } \\
\text { SRAS-CoV-2 } \\
(n=127)\end{array}$} & \multicolumn{2}{|c|}{$\begin{array}{l}\text { Tous les patients } \\
\qquad(n=269)\end{array}$} \\
\hline & $\mathbf{n}$ & $\%$ & $\mathbf{n}$ & $\%$ & n & $\%$ & $\mathbf{n}$ & $\%$ \\
\hline \multicolumn{9}{|c|}{ Catégorie d'âge (années) (suite) } \\
\hline 5 à 9 & 46 & 36 & 51 & 36 & 30 & 24 & 81 & 30 \\
\hline 10 à 14 & 30 & 24 & 34 & 24 & 15 & 12 & 49 & 18 \\
\hline 15 à 19 & 14 & 11 & 15 & 11 & 12 & 9 & 27 & 10 \\
\hline \multicolumn{9}{|l|}{ Intervalle } \\
\hline $\begin{array}{l}\text { Âge médian } \\
\text { (intervalle en } \\
\text { années) }\end{array}$ & & $8(0-18)$ & & $8(0-18)$ & & $4(0-17)$ & & $6(0-18)$ \\
\hline \multicolumn{9}{|l|}{ Hospitalisation ${ }^{c}$} \\
\hline Oui & 127 & 100 & 142 & 100 & 125 & 98 & 267 & 99 \\
\hline Non & 0 & 0 & 0 & 0 & 2 & 2 & 2 & 1 \\
\hline \multicolumn{9}{|c|}{ Admission aux soins intensifs } \\
\hline Oui & 98 & 57 & 76 & 54 & 22 & 17 & 98 & 36 \\
\hline Non & 54 & 43 & 66 & 46 & 102 & 80 & 168 & 62 \\
\hline Inconnu & 0 & 0 & 0 & 0 & 3 & 2 & 3 & 1 \\
\hline \multicolumn{9}{|l|}{ Résultat $^{d}$} \\
\hline Rétablissement & 72 & 57 & 82 & 58 & 102 & 80 & 184 & 68 \\
\hline $\begin{array}{l}\text { Convalescence/ } \\
\text { stabilité }\end{array}$ & 53 & 42 & 58 & 41 & 22 & 17 & 80 & 30 \\
\hline Détérioration & 1 & 1 & 1 & 1 & 0 & 0 & 1 & 0 \\
\hline Inconnu & 1 & 1 & 1 & 1 & 3 & 2 & 4 & 1 \\
\hline
\end{tabular}

Abréviation : SRAS-CoV-2, coronavirus du syndrome respiratoire aigu sévère 2

a Coronavirus du syndrome respiratoire aigu sévère 2

${ }^{b}$ Les pourcentages sont arrondis au nombre entier le plus proche. La somme des pourcentages d'une catégorie peut donc ne pas être égale à $100 \%$

' Une province ou un territoire n'a signalé que les cas hospitalisés

${ }^{d}$ Les résultats des patients ont été mis à jour par les autorités sanitaires provinciales et territoriales lorsque cela était possible. Les données présentées ici étaient celles de la dernière mise à jour

À ce jour, le nombre de cas de MIS-C signalés au Canada a été le plus élevé de décembre 2020 à début mars 2021. Cela faisait suite à un pic de l'incidence de la COVID-19 signalée chez les enfants et les jeunes en décembre 2020 et début janvier 2021 (figure 1). Bien que l'incidence de la COVID-19 ait diminué entre janvier 2021 et début mars 2021, le nombre de cas de MIS-C signalés est resté élevé pour plusieurs raisons. Premièrement, le MIS-C est un syndrome post-viral et les rapports de la littérature suggèrent qu'il se manifeste généralement 2 à 6 semaines après I'infection par le SRAS-CoV-2 (5-7). On s'attend donc à ce que le nombre de cas reste élevé dans les semaines qui suivent une forte incidence de COVID-19. Deuxièmement, le nombre de cas de COVID-19 chez les enfants et les jeunes au Canada était encore élevé au cours des mois de février et mars 2021. Comme les cas de COVID-19 chez les enfants et les jeunes ont à nouveau augmenté fin mars et en avril 2021, nous pouvons nous attendre à voir des cas supplémentaires de MIS-C signalés après ces périodes.

\section{Forces et faiblesses}

Les données contenues dans ce rapport sont soumises à plusieurs limites. Premièrement, les données sont incomplètes, car les provinces et les territoires n'ont pas tous participé à la surveillance nationale du MIS-C et une province n'a signalé que les cas confirmés en laboratoire. Deuxièmement, la déclaration des cas peut également être retardée en raison de la capacité limitée des autorités sanitaires provinciales et territoriales. Les chiffres liés aux cas pour les deux derniers mois en particulier doivent être interprétés avec prudence. Troisièmement, il est difficile de discerner si les cas ont été infectés par la COVID-19 ou non en raison de plusieurs facteurs : le test RT-PCR peut être négatif s'il est effectué trop tard dans l'évolution de l'infection; le test sérologique peut ne pas être disponible; l'interprétation des résultats sérologiques présente des difficultés inhérentes; et les patients peuvent ne pas savoir qu'ils ont été en contact avec un cas de COVID-19. Pour cette raison, les cas sans preuve connue d'infection par le SRAS-CoV-2 ou d'exposition à un cas 
Figure 1 : Cas déclarés de syndrome inflammatoire multisystémique chez les enfants, par semaine épidémiologique d'apparition des symptômes, comparés aux cas de COVID-19 chez les enfants et les jeunes de 0 à 19 ans, Canada, du 11 mars 2020 au 2 octobre $2021(N=269)$

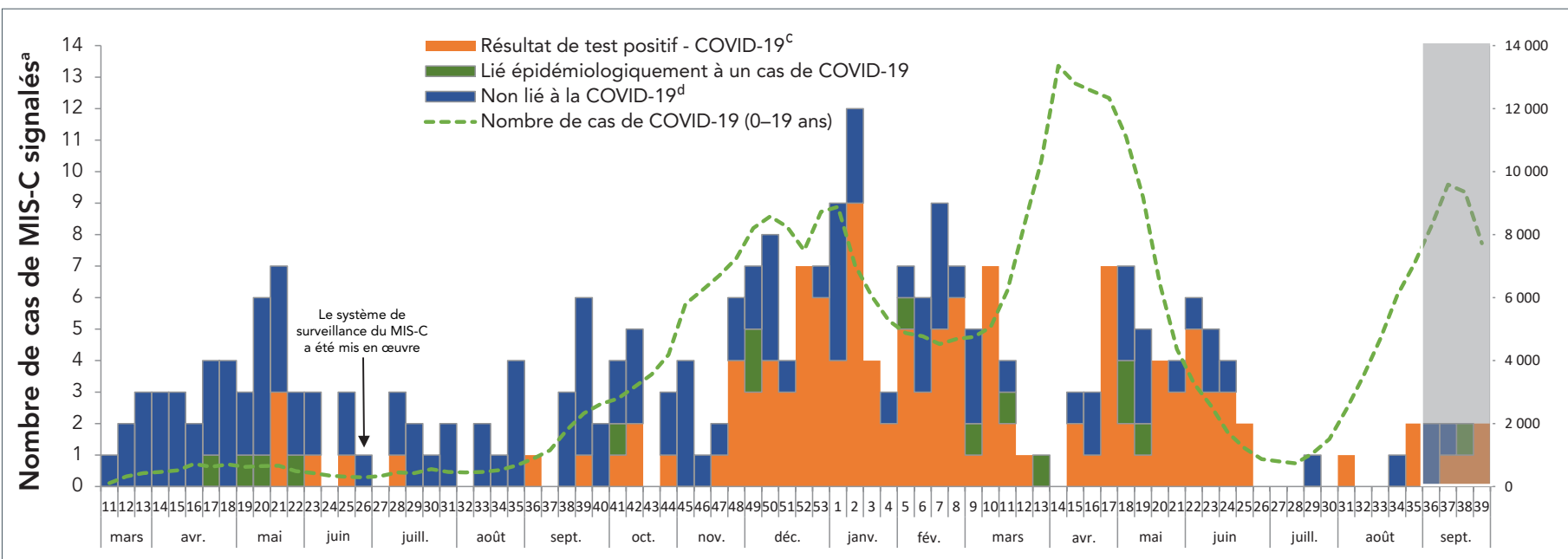

Semaine épidémiologique d'apparition des symptômes

Abréviations : COVID-19, maladie à coronavirus 2019; MIS-C, syndrome inflammatoire multisystémique chez les enfants

Source de données : Déclaration nationale des provinces et territoires de MIS-C

b Source des données : Système national de surveillance des maladies à coronavirus 2019

Résultat de test de dépistage de la COVID-19 positif par RT-PCR, test d'antigène ou sérologie

Aucune preuve connue d'infection par le SRAS-CoV-2 ou d'exposition à un cas de COVID-19

Note : La zone ombragée représente une période pendant laquelle on s'attend à ce que des cas se soient produits, mais n'aient pas encore été signalés au niveau national

de COVID-19 ont été inclus dans l'analyse; cependant, ces cas peuvent ne pas être liés à la COVID-19 et, par conséquent, ne pas être de véritables cas de MIS-C. En raison des similitudes entre les symptômes du MIS-C et de la maladie de Kawasaki et des difficultés à diagnostiquer ces maladies, il peut y avoir une mauvaise classification des cas, en particulier des cas sans lien connu avec la COVID-19. Des données plus détaillées sur les tests de laboratoire sont nécessaires pour mieux différencier les cas liés et non liés à la COVID-19.

\section{Conclusion}

Les cas de MIS-C au Canada sont rares; cependant, lorsque la maladie survient, elle est grave, presque tous les cas nécessitant une hospitalisation et un tiers une admission à l'unité de soins intensifs. Tous les enfants canadiens atteints de MIS-C se sont rétablis ou se rétablissent, sans qu'aucun décès ne soit signalé. La tendance temporelle du MIS-C s'aligne sur celle de l'incidence de la COVID-19 chez les enfants, avec un décalage de deux à six semaines. Ce schéma a été rapporté dans d'autres publications, ce qui confirme 1) I'association temporelle du MIS-C avec la COVID-19 et 2) l'idée actuelle selon laquelle le MIS-C résulte d'une réponse immunologique retardée à l'infection par le SRAS-CoV-2 (7). Au Canada, le MIS-C est plus susceptible de se produire chez les garçons que chez les filles.

Bien que le MIS-C soit rare, il est grave, et on ne sait pas encore pourquoi certains enfants développent ce syndrome et d'autres pas. En outre, les effets à long terme du MIS-C restent largement inconnus. Le moyen le plus efficace de prévenir les cas de maladie grave chez les enfants est de suivre les mesures de santé publique qui empêchent la propagation de la COVID-19, notamment la distance physique, le port de masques, I'hygiène des mains, rester à la maison quand on est malade et de se faire vacciner contre la COVID-19 quand on y a droit. L'Agence de la santé publique du Canada continuera de travailler avec ses partenaires provinciaux et territoriaux pour surveiller les cas de maladies inflammatoires graves chez les enfants et tenir le public informé du risque pour les enfants et les jeunes.

\section{Déclaration des auteurs}

M. L. - Méthodologie, logiciel, analyse formelle, enquête, conservation des données, rédaction-version originale, rédaction-révision et édition, visualisation

M. S. - Conceptualisation, rédaction-version originale, rédaction-révision et édition

S. G. S. - Conceptualisation, rédaction-version originale, rédaction-révision et édition

M. A. - Rédaction-version originale, rédaction-révision et édition

L. E. - Rédaction-version originale, rédaction-révision et édition

S. L. - Rédaction-version originale, rédaction-révision et édition

A. D. C. - Rédaction-version originale, rédaction-révision et édition

Y. A. L. - Conceptualisation, méthodologie, rédaction-version originale, rédaction-révision et édition, administration du projet

\section{Intérêts concurrents}

Aucun. 


\section{Remerciements}

Nous tenons à remercier toutes les autorités sanitaires provinciales et territoriales et les collaborateurs du Programme canadien de surveillance pédiatrique pour leur contribution à la surveillance du syndrome inflammatoire multisystémique chez les enfants (MIS-C) au Canada. Nous tenons également à remercier nos collègues de l'Agence de la santé publique du Canada, T. Bennett, pour la validation des données, ainsi que R. MacTavish et A. Agarwal pour la gestion de la base de données et la mise à jour du présent rapport.

\section{Financement}

Aucun

\section{Références}

1. Centers for Disease Control and Prevention. CDC Health Alert Network. Multisystem Inflammatory Syndrome in Children (MIS-C) Associated with Coronavirus Disease 2019 (COVID-19). Atlanta, GA: CDC; 2020.

https://emergency.cdc.gov/han/2020/han00432.asp

2. Word Health Organization. Multisystem inflammatory syndrome in children and adolescents temporally related to COVID-19. Geneva, CH: WHO; 2020.

https://www.who.int/news-room/commentaries/detail/ multisystem-inflammatory-syndrome-in-children-andadolescents-with-covid-19

3. Kucirka LM, Lauer SA, Laeyendecker O, Boon D, Lessler J. Variation in False-Negative Rate of Reverse Transcriptase Polymerase Chain Reaction-Based SARS-CoV-2 Tests by Time Since Exposure. Ann Intern Med 2020;173(4):262-7. DOI PubMed
4. Centers for Disease Control and Prevention. About Kawasaki Disease. Atlanta, GA: CDC; 2020 (accédé 2021-04-07). https://www.cdc.gov/kawasaki/about.html

5. Feldstein LR, Rose EB, Horwitz SM, Collins JP, Newhams MM, Son MB, Newburger JW, Kleinman LC, Heidemann SM, Martin AA, Singh AR, Li S, Tarquinio KM, Jaggi P, Oster ME, Zackai SP, Gillen J, Ratner AJ, Walsh RF, Fitzgerald JC, Keenaghan MA, Alharash H, Doymaz S, Clouser KN, Giuliano JS Jr, Gupta A, Parker RM, Maddux AB, Havalad V, Ramsingh S, Bukulmez $H$, Bradford TT, Smith LS, Tenforde MW, Carroll CL, Riggs BJ, Gertz SJ, Daube A, Lansell A, Coronado Munoz A, Hobbs CV, Marohn KL, Halasa NB, Patel MM, Randolph AG; Overcoming COVID-19 Investigators; CDC COVID-19 Response Team. Multisystem Inflammatory Syndrome in U.S. Children and Adolescents. N Engl J Med 2020;383(4):334-46. DOI PubMed

6. Dufort EM, Koumans EH, Chow EJ, Rosenthal EM, Muse A, Rowlands J, Barranco MA, Maxted AM, Rosenberg ES, Easton D, Udo T, Kumar J, Pulver W, Smith L, Hutton B, Blog D, Zucker H; New York State and Centers for Disease Control and Prevention Multisystem Inflammatory Syndrome in Children Investigation Team. Multisystem Inflammatory Syndrome in Children in New York State. N Engl J Med 2020;383(4):347-58. DOI PubMed

7. Belay ED, Abrams J, Oster ME, Giovanni J, Pierce T, Meng L, Prezzato E, Balachandran N, Openshaw JJ, Rosen HE, Kim M, Richardson G, Hand J, Tobin-D'Angelo M, Wilson S, Hartley A, Jones C, Kolsin J, Mohamed H, Colles Z, Hammett T, Patel P, Stierman B, Campbell AP, Godfred-Cato S. Trends in Geographic and Temporal Distribution of US Children With Multisystem Inflammatory Syndrome During the COVID-19 Pandemic. JAMA Pediatr 2021;175(8):837-45. DOI PubMed 